\title{
Early Biochemical Changes in the Embryonic Rat Heart after Teratogen Treatment ${ }^{1,2}$
}

\author{
DENNIS O. OVERMAN ${ }^{3}$ AND ALLAN R. BEAUDOIN \\ Department of Anatomy, The University of Michigan, \\ Ann Arbor, Michigan 48104
}

\begin{abstract}
The effects on mucopolysaccharide synthesis in isolated embryonic rat hearts of in vivo and/or in vitro 6-aminonicotinamide, dietary-induced folic acid deficiency, aminopterin, and trypan blue were investigated using uptake of ${ }^{35} \mathrm{~S}$-sodium sulfate to measure the rate of mucopolysaccharide synthesis. Intraperitoneal injection of 6-aminonicotinamide on day 11 of gestation caused inhibition of mucopolysaccharide synthesis which was most pronounced at day 13. A similar effect followed in vitro 6-aminonicotinamide treatment. Maternal folic acid deficiency on days 9-11 of pregnancy caused decreased mucopolysaccharide synthesis in embryonic hearts at day 14 . In vitro treatment with the folic acid antagonist aminopterin inhibited the rate of synthesis on days 13-16. In vivo trypan blue on day 9 of pregnancy had no effect on the rate of mucopolysaccharide synthesis. However, in vitro trypan blue treatment of embryonic hearts resulted in a marked depression in synthetic rate. Aminopterin and 6-aminonicotinamide had no noticeable effect on the rate of protein synthesis in isolated embryonic hearts as determined by measurement of ${ }^{3} \mathrm{H}$-leucine incorporation. Thus mucopolysaccharide synthesis in isolated embryonic rat hearts was susceptible to the action of the teratogens used. It is postulated that these teratogens act by blocking a biochemical reaction in the synthesis of mucopolysaccharides and are not affecting the cellular machinery necessary for general protein synthesis.
\end{abstract}

Present knowledge of the mechanisms of action of teratogens is very limited, even in laboratory animals. It may be that many teratogens act by means of an effect on some of the large molecules important in development. Information on mechanisms of action may be provided by investigating some of the early biochemical changes in developing systems after teratogen treatment.

Mucopolysaccharides of the embryonic rat heart were selected for biochemical investigation in this study. In the heart, mucopolysaccharides are found in the endocardial cushions, interatrial septum, bulbus and large blood vessels, and cardiac jelly (Walker, '61). Barry ('48) and Patton et al. ('48) described the role of the cardiac jelly as twofold: allowing the heart to function as a pump while changing shape and position during development, and serving as a substrate for the molding action of the bloodstream in shaping the heart. The gelatinous property of mucopolysaccharides is thought to be related to these roles of the cardiac jelly (Gessner and Boström, '65; Gessner et al., '65).

Recent evidence has linked both inherited and experimentally produced congenital malformations to decreased mucopolysaccharide synthesis. Mathews ('67) observed decreased sulfated mucopolysaccharide in the limbs of chick embryos with inherited lethal skeletal defects. Meyer ('69) suggested that these defects may be the result of deletions of some biosynthetic enzyme systems leading to mucopolysaccharide synthesis. Teratogens have produced abnormal mucopolysaccharide synthesis in vitro (Beaudoin, Boström,

1 From a dissertation submitted to the Horace $H$ Rackham School of Graduate Studies of The University of Michigan in partial fulfilment of the requiresity of Michigan in partial fulfilment of the

2 Supported by NIH grants GM00312 and HDo0400.

3 Present address: Department of Molecular, Cellular and Developmental Biology, University of Colorado, Boulder, Colorado $\mathbf{8 0 3 0 2}$ 
Friberg and Larsson, '69; Beaudoin, Boström, Larsson and Lohmander, '69) and in vivo (Kochhar et al., '67, '68). In spite of these indications that mucopolysaccharides are important in prenatal development no evidence exists that they play a role in cardiac teratogenesis; but because of their presence in the cardiac jelly at a critical stage, the question was investigated.

Teratogens were used that have produced cardiovascular malformations: 6aminonicotinamide (6-AN), folic acid deficiency, aminopterin, and trypan blue (Nelson et al., '55; Chamberlain and Nelson, '63; Fox and Goss, '56, '57, '58; Wilson, '60; Kinney and Morse, '64). A preliminary study demonstrated that the teratogens chosen cause malformations of the heart in the stock of rats used.

\section{MATERIALS AND METHODS}

Animals. Virgin female Wistar rats (Albino Farms, Red Bank, New Jersey) were used. They were fed Purina Lab Chow ad libitum, except when receiving experimental diets, and were given water ad libitum. One male of the same stock was caged with six females overnight, and the day of finding sperm was designated day 1 of pregnancy.

In vivo treatment. Single ip injections of $6 \mathrm{mg} / \mathrm{kg} 6-\mathrm{AN}$ were given on day 11 of pregnancy. A folic acid-deficient diet ${ }^{4}$ was fed on days 9-11. Trypan blue in a 2\% aqueous solution was administered as single ip injections of $140 \mathrm{mg} / \mathrm{kg}$ on day 9.

Recovery and incubation of hearts. The uterus was excised on days 11-21 and placed in a Petri dish of Tyrode's solution. The embryos were transferred to a second dish of Tyrode's solution where the hearts were removed and placed in a 20-ml Erlenmeyer flask containing $2 \mathrm{ml}$ of Tyrode's solution. One to four hearts were placed in each flask and the flasks were positioned in a shaker water bath at $37^{\circ} \mathrm{C}$ and gently agitated during incubation. Each flask was individually aerated with a mixture of $5 \%$ $\mathrm{CO}_{2}$ in oxygen. Hearts were incubated for either 2 or 3 hours before isotope was added to the incubation medium.

In vitro treatment. Control hearts of 11-21 days gestation age were incubated in Tyrode's solution for 1 hour before the teratogen was added. 6-AN was added to the incubation medium in a final concentration of $1 \mathrm{~mm}$, aminopterin in a concentration of $2 \mathrm{~mm}$, and trypan blue in a final concentration of 0.6 or $1 \mathrm{~mm}$.

Folic acid was added in a concentration of $1 \mathrm{~mm}$ to a group of experimental hearts from folic acid-deficient mothers, as well as a group of paired controls of ages 11-21 days.

Isotope administration. ${ }^{35} \mathrm{~S}$-sodium sulfate $^{5}$ was added in a concentration of $10 \mu \mathrm{Ci} / \mathrm{ml}$ of incubation medium. After 1 or 2 hours isotope uptake was stopped by adding $1 \mathrm{ml}$ of $2 \%$ monoiodoacetic acid. Ten minutes later the fluid was drawn off and replaced with a saturated solution of sodium sulfate. Hearts were left overnight in this solution, then rinsed once with distilled water.

6-AN and aminopterin were tested in vitro for their effects on the uptake of labeled leucine as an indication of the rate of protein synthesis during incubation. Hearts were incubated in the presence of the teratogen for 2 hours before ${ }^{3} \mathrm{H}$-leucine $(2.5 \mu \mathrm{Ci}$ /flask) was added. Two hours later uptake was stopped by adding monoiodoacetic acid; hearts were left in distilled water overnight and rinsed once again with distilled water the following morning.

Scintillation counting. After being washed and rinsed, hearts were blotted on filter paper, weighed on a Cahn Electrobalance, and placed in scintillation vials. Tissue solubilizer ${ }^{6}(0.5 \mathrm{ml})$ was added to each vial and the hearts were allowed to become solubilized at room temperature for 1 hour to overnight as necessary. After solubilization $14.5 \mathrm{ml}$ of the scintillator" was added to each vial. The samples were counted in a Packard Tri-Carb liquid scintillation spectrometer. The rate of mucopolysaccharide synthesis per milligram wet weight of tissue was calculated as a function of disintegrations per minute (dpm) of measured isotope decay, correcting for individual sample quenching and for isotope decay.

\footnotetext{
4 General Biochemicals, Chagrin Falls, Ohio.

5 New England Nuclear, Boston.

6 Soluene, Packard Instrument Company, Inc., Chicago.

7 Omnifiuor, New England Nuclear, Boston.
} 
Autoradiography and histochemistry. Histochemical and autoradiographic studies were performed on young from at least one experimental and one control litter of gestation days 11 through 21. After exposure to ${ }^{35} \mathrm{~S}$-sulfate hearts were prepared for autoradiography by the liquid emulsion dipping method of Gude ('68). Sectioned and mounted hearts were stained with toluidine blue, alcian blue, or aldehyde fuchsin to determine the location of mucopolysaccharides.

Each experiment involved experimental and control hearts processed side by side and incubated simultaneously. Routine histological examination was done on representative hearts from each experiment in order to compare heart morphology before and after incubation.

\section{RESULTS}

Control pattern of ${ }^{85}$ S-sulfate uptake. The rate of mucopolysaccharide synthesis in control hearts expressed as $\mathrm{dpm} / \mathrm{mg}$ wet weight is shown in figure 1 . Beginning with day 11 there was a rapid increase in the rate of synthesis with advancing age, reaching a peak at day 14 , after which it decreased.

$6-A N$ in vivo. Hearts recovered from embryos of females treated with 6-AN on day 11 showed inhibition in the rate of sulfate uptake at younger ages (days 13-
15: $P<0.01$ ), which disappeared by day 16 (fig. 1).

6-AN in vitro. Effects of in vitro 6-AN were similar to those of in vivo treatment: early inhibition of mucopolysaccharide synthesis disappearing by day 16 (days 13-15: $P<0.01$, fig. 1). Figure 2 expresses the experimental results as a percentage of the rate of synthesis in the control hearts and more graphically shows the inhibitory effect of the teratogen. The rate of protein synthesis as measured by incorporation of ${ }^{3} \mathrm{H}$-leucine by day- 13 hearts was unaffected by in vitro 6-AN.

Folic acid deficiency. The effect of maternal dietary folic acid deficiency on mucopolysaccharide synthesis in the embryonic hearts is indicated in figure 3 . The change in the scale of the ordinate reflects a longer time of exposure to the isotope in this experiment. The inhibition in the rate of sulfate uptake by the day-14 hearts (approximately 25\%) was statistically significant $(P<0.01)$.

The results indicate that adding folic acid in vitro to control hearts had no effect on the rate of sulfate uptake. However, when folic acid was added to the experimental hearts the inhibition due to folic acid deficiency was eliminated (fig. 3).

Aminopterin in vitro. Aminopterin treatment in vitro caused inhibition of sulfate uptake on days 13-16 $(P<0.01)$ after which the effect disappeared (fig. 4). Pro-

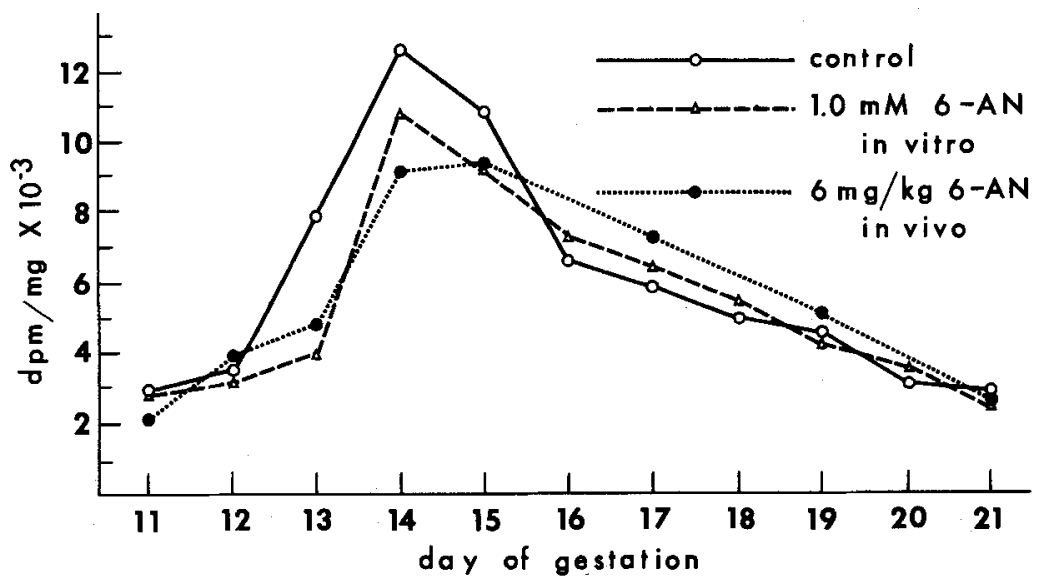

Fig. 1 Effects of in vivo and in vitro 6-aminonicotinamide (6-AN) on the rate of mucopolysaccharide biosynthesis in the prenatal rat heart. $P<0.01$ on days $13-15$ for both in vivo and in vitro experiments. Each point represents the mean for several embryos from at least three litters. Time of isotope incorporation: 1 hour. 


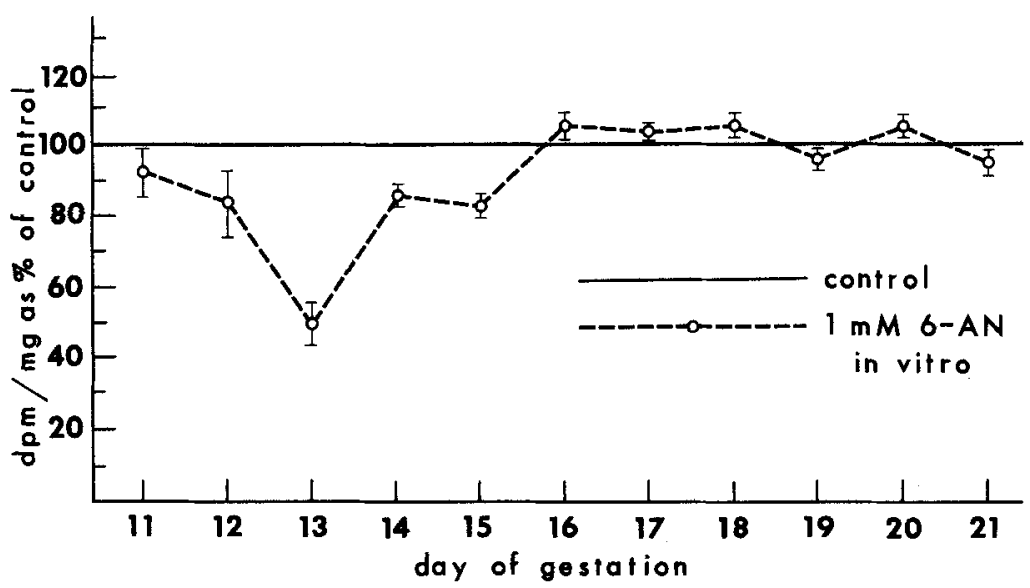

Fig. 2 Effects of in vitro 6-aminonicotinamide on the rate of mucopolysaccharide biosynthesis in the prenatal rat heart expressed as percentage of the control rate. Vertical bars represent standard error of the mean. Each point represents the mean for several embryos from at least three litters. Time of isotope incorporation: 1 hour.

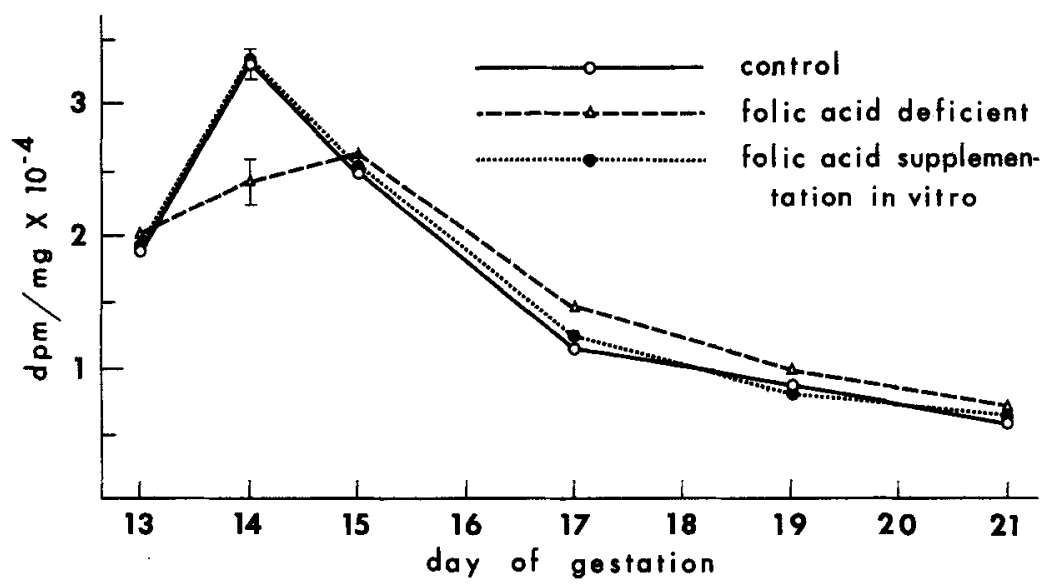

Fig. 3 Effects of maternal folic acid deficiency and in vitro folic acid supplementation on the rate of mucopolysaccharide biosynthesis in the prenatal rat heart. Vertical bars represent standard error of the mean. Each point represents the mean for several embryos from at least three litters. Time of isotope incorporation: 2 hours.

tein synthesis was not significantly affected (fig. 5).

Trypan blue in vivo. Treatment of pregnant mothers with trypan blue had no noticeable effect on the synthesis of mucopolysaccharides in the offspring hearts (fig. 6).

Trypan blue in vitro. The inhibition of mucopolysaccharide synthesis by $1 \mathrm{~mm}$ trypan blue in vitro was so marked (fig. 6) that it was difficult to determine at what time the greatest effect occurred. Gross inspection of the hearts after 4 hours of in- cubation in trypan blue showed that they still beat spontaneously.

Administration of $0.6 \mathrm{~mm}$ trypan blue in vitro also had a profound effect, but the shape of the curve obtained was more like that of the other treatments described (fig. 6). Inhibition of mucopolysaccharide synthesis after in vitro trypan blue was apparent in prenatal hearts of all ages tested.

Autoradiography and histochemistry. Granules were found scattered throughout the embryonic heart tissue, but large con- 


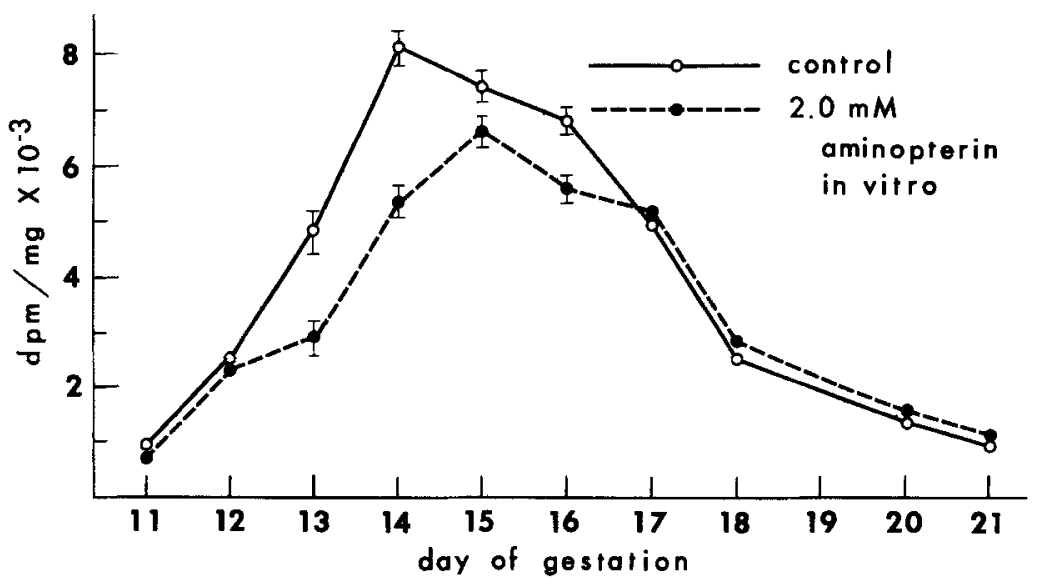

Fig. 4 Effects of in vitro aminopterin on the rate of mucopolysaccharide biosynthesis in the prenatal rat heart. Vertical bars represent standard error of the mean. Each point represents the mean for several embryos from at least three litters. Time of isotope incorporation: 2 hours.

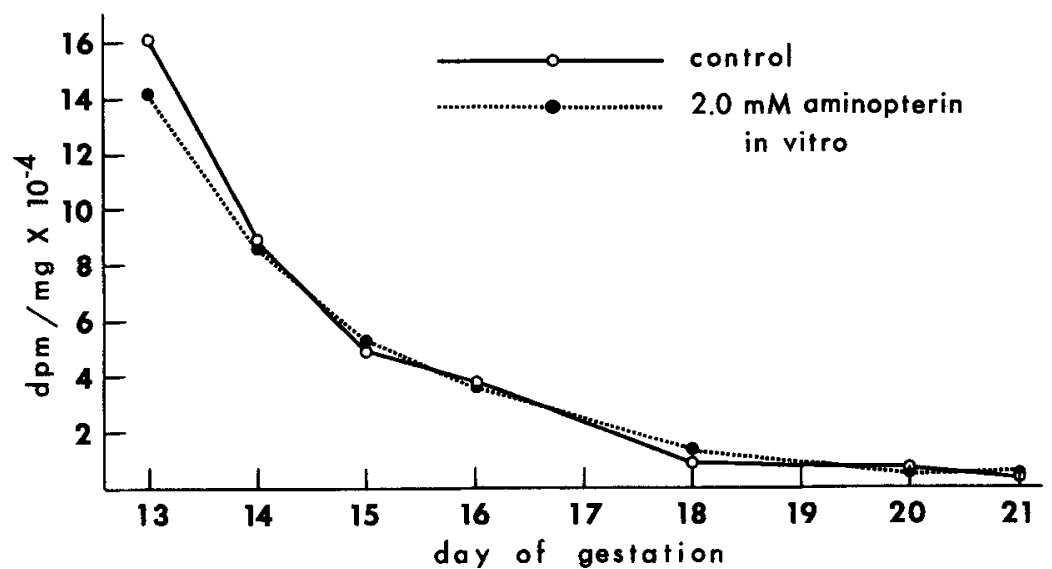

Fig. 5 Effects of in vitro aminopterin on the rate of incorporation of tritiated leucine as an indicator of the rate of protein synthesis in prenatal rat hearts. Each point represents the mean for several embryos from at least three litters. Time of isotope incorporation: 2 hours.

centrations were localized in the atrioventricular area, the interventricular septal area, and the partitioning truncus.

Examination of hematoxylin-and-eosinstained hearts before and after incubation revealed no observable difference in heart morphology. Histochemical tests localized mucopolysaccharides in the same general areas where autoradiography indicated the presence of ${ }^{35} \mathrm{~S}$, namely the atrioventricular area, interventricular septum, and truncus.

\section{DISCUSSION}

This investigation showed that isolated embryonic rat hearts incorporated measurable amounts of ${ }^{35} \mathrm{~S}$-sulfate, as did isolated chick embryo hearts studied by Gessner et al. ('65). Gessner and Boström ('65) reported that incorporation of sulfate per unit weight by the isolated chick heart is inversely proportional to the weight of the heart, and therefore to the age of the embryo. They explained this observation by 


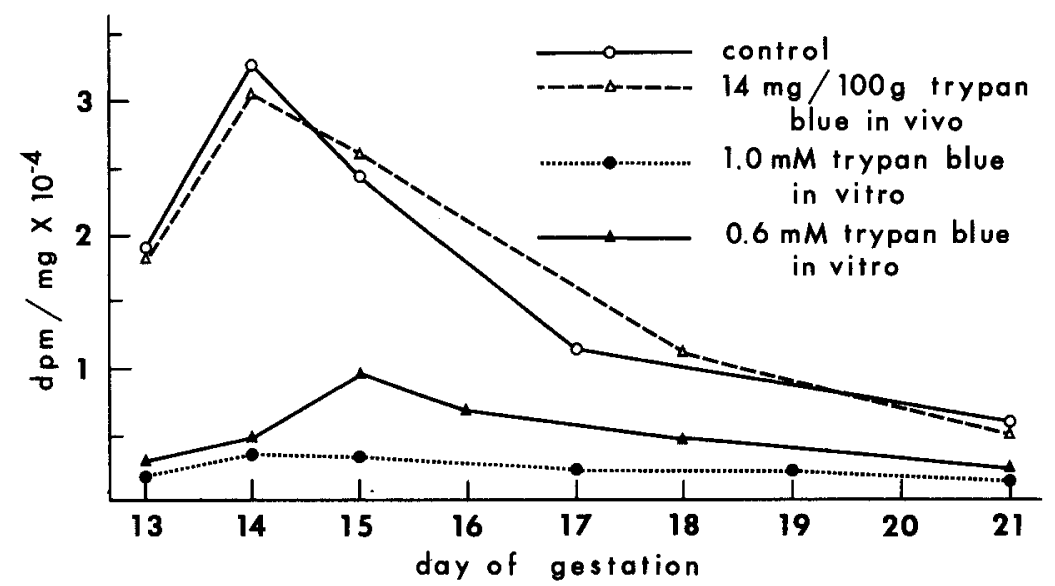

Fig. 6 Effects of in vivo and in vitro trypan blue on the rate of mucopolysaccharide biosynthesis in the prenatal rat heart. Each point represents the mean for several embryos from at least three litters. Time of isotope incorporation: 2 hours.

the fact that the cardiac jelly makes up a proportionately larger part of the younger heart. Thus the amount of cardiac jelly can be directly related to the pattern of uptake of labeled sulfate by the isolated heart. The present study revealed that the incorporation of ${ }^{35} \mathrm{~S}$-sulfate per milligram of heart tissue by the rat heart was also inversely proportional to the age of the embryo after day 14 , at which time the active processes of organogenesis in the rat heart are complete and the quantity of cardiac jelly has begun to decrease.

In vivo 6 -AN produced an inhibitory effect on sulfate uptake in embryonic hearts during days 13-15. In the present experiment the teratogen had its maximum effect on day 13 , which may represent the most active period of production of cardiac jelly and possibly the day of most active involvement of the cardiac jelly in the organogenesis of the heart. The ineffectiveness of in vivo 6-AN after day 15 indicated the end of the susceptible period of the heart to this teratogen. When $6-\mathrm{AN}$ was given to pregnant females on day 11 its greatest effect on the mucopolysaccharide synthesis of the heart still appeared on day 13. This indicates that there is a delay between the time of administration of the drug and the appearance of measurable effects. Chamberlain and Nelson ('63) found no heart malformations when 6-AN was given after day 12 . It may be that during this delay between administration of the teratogen and appearance of the effects the heart's susceptible period for malformations related to mucopolysaccharide synthesis had passed.

The close similarity between the effects of 6-AN in vivo and in vitro is not without precedent, as Turbow and Chamberlain ('68) have described in the case of the whole rat embryo in vitro. Study of incorporation of ${ }^{3} \mathrm{H}$-leucine in the presence of in vitro 6-AN on day 13 , the day of maximum effect of the teratogen, showed that the heart's ability to synthesize protein was not affected. Therefore the cellular mechanism for the assembly of proteins was probably not damaged by the treatment, and some of these same cellular components are active in the sulfation and assembly of mucopolysaccharides. This finding suggests that 6-AN selectively inhibits mucopolysaccharide synthesis.

Whether any influence of 6-AN on mucopolysaccharide synthesis is due to its role as an antimetabolite of niacin is a matter of some speculation. Landauer and Clark ('62) stated that the teratogenic effects of 6-AN are not necessarily related to its properties as an antimetabolite, and therefore not necessarily related to niacin metabolism. However, since the present results with 6-AN suggest that its action is related to metabolism, it is speculated that niacin metabolism is involved. This may be through the formation of a 6amino analogue of NAD which could ad- 
versely affect the action of NAD-dependent enzyme systems. The process of incubation itself resulted in no noticeable morphologic effects on the heart tissue, as revealed by histological inspection of hearts before and after a 4-hour incubation period in 6-AN.

Cardiac defects produced by a folic aciddeficient diet were most pronounced when the diet was given on days 9-11 of gestation (Nelson et al., '52, '55; Baird et al., '54). The same experimental procedure in this study caused inhibition of the synthesis of mucopolysaccharides at only one time, day 14. A possible explanation for this finding may be based on a proposed lag period of about 48 hours between administration of a teratogen and appearance of its effects (Runner, '65). After day 14 of gestation the cardiac jelly of the heart appears to be decreasing; the importance of this substance in terms of sulfate uptake has already been discussed. Therefore only a brief period during development exists for effects of maternal folic acid deficiency on cardiac mucopolysaccharide synthesis to appear.

Treatment of embryonic hearts in vitro with the folic acid antagonist aminopterin provided a method for studying folic acid deficiency in greater detail under more controlled conditions than was possible using a deficient diet, since the dose of the drug was accurately controlled, and the time of exposure of the hearts to the treatment precisely determined. It was found that aminopterin produced an immediate measurable effect on the rate of sulfate uptake by the embryonic hearts on days 13-16. But leucine-uptake experiments showed that the drug had no noticeable effect on the ability of the hearts to synthesize proteins.

Supplementation with folic acid showed that the mechanism for the utilization of sulfates in the synthesis of mucopolysaccharides was not destroyed, confirming the interpretation of the results of the leucineuptake experiments. Therefore it appears that the teratogens used in this study influence the heart by means of a metabolic route rather than by damaging the cellular capacity for general protein synthesis.

Kochhar et al. ('68) observed that maternal treatment with trypan blue stimulated rather than inhibited uptake of ${ }^{35} \mathrm{~S}$ - sulfate by embryonic mouse tissues. In the present experiment in vivo trypan blue had no measurable effect on mucopolysaccharide synthesis in the isolated embryonic rat heart. It must be noted that Kochhar et al. ('68) measured ${ }^{35} \mathrm{~S}$-sulfate incorporation by whole embryos rather than isolated organs. The discrepancy between their findings and those of the present study might be due to the different species used or might reflect a stimulation of sulfate uptake in some organ or system other than the heart. In vitro trypan blue on the other hand had a most profound effect on isotope uptake by the embryonic hearts. A first impression upon examination of these results was that the presence of trypan blue in the incubation medium had killed the hearts before they had a chance to incorporate a significant amount of the isotope. However, direct observation indicated that the hearts were still alive and beating spontaneously following the treatment.

It is obvious from this and other studies that not all hearts were destined to be malformed by the teratogens, although very few of the hearts studied failed to show inhibition of mucopolysaccharide synthesis during the susceptible stages in heart morphogenesis. Once again the problem arises as to what unknown factors operate during the period of development just after teratogen treatment to protect some embryos and enable them to recover and develop normally. Thus while all hearts may be affected by the teratogen treatment, as reflected by their lowered rate of mucopolysaccharide synthesis, the lowered rate of synthesis is only one factor in abnormal development.

\section{LITERATURE CITED}

Baird, C. D., M. M. Nelson, I. W. Monie and H. M. Evans 1954 Congenital cardiovascular anomalies induced by pteroylglutamic acid deficiency during gestation in the rat. Circ. Res., 2: 544-554.

Barry, A. 1948 The functional significance of the cardiac jelly in the tubular heart of the chick embryo. Anat. Rec., 102: 289-298.

Beaudoin, A. R., H. Boström, U. Friberg and K. S. Larsson 1969 The effect of sodium salicylate on the glutamine- and glucosamine-induced stimulation of $\mathbf{S}^{35}$-sulfate incorporation in vitro. Ark. Kemi, 30: 523-527.

Beaudoin, A. R., H. Boström, K. S. Larsson and S. Lohmander 1969 The influence of in vivo 
trypan blue and prednisolone acetate treatment upon $\mathrm{S}^{35}$-sulfate in vitro incorporation in cartilage. Ark. Kemi, 31: 1-4.

Chamberlain, J. G., and M. M. Nelson 1963 Congenital abnormalities in the rat resulting from single injections of 6-aminonicotinamide during pregnancy. J. Exp. Zool., 153: 285-300.

Fox, M. H., and C. M. Goss 1956 Experimental production of a syndrome of cardiovascular defects in rats. Anat. Rec., 124: 189-208.

- 1957 Experimentally produced malformations of the heart and great vessels in rat fetuses. Atrial and caval abnormalities. Anat. Rec., 129: 309-332.

- 1958 Experimentally produced malformations of the heart and great vessels in rat fetuses. Transposition complexes and aortic arch abnormalities. Am. J. Anat., 102: 65-92.

Gessner, I. H., and H. Boström 1965 In vitro studies on ${ }^{35} \mathrm{~S}$-sulfate incorporation into the acid mucopolysaccharides of chick embryo cardiac jelly. J. Exp. Zool., 160: 283-290.

Gessner, I. H., A. E. Lorincz and H. Boström 1965 Acid mucopolysaccharide content of the cardiac jelly of the chick embryo. J. Exp. Zool., 160; 291-298.

Gude, W. D. 1968 Autoradiographic Techniques. Localization of Radioisotopes in Biological Material. Prentice Hall, Englewood Cliffs, New Jersey.

Kinney, C. S., and L. M. Morse 1964 Effect of a folic acid antagonist, aminopterin, on fetal development and nucleic acid metabolism in the rat. J. Nutr., 84: 288-294.

Kochhar, D. M., H. Boström, K. S. Larsson and L. Reio 1967 Influence of trypan blue and related compounds on ${ }^{35} \mathrm{~S}$-sulfate metabolism of cartilage in vitro. Eur. J. Pharmacol., 1: 326-333.

Kochhar, D. M., K. S. Larsson and H. Boström 1968 Embryonic uptake of $\mathrm{S}^{35}$-sulfate: change in level following treatment with some teratogenic agents. Biol. Neonat., 12:41-53.

Landauer, W., and E. M. Clark 1962 The interaction in teratogenic activity of the two niacin analogs 3-acetylpyridine and 6-aminonicotinamide. J. Exp. Zool., 151: 253-258.

Mathews, M. B. 1967 Chondroitin sulphate and collagen in inherited skeletal defects of chickens. Nature, 213: 1255-1256.

Meyer, K. 1969 Biochemistry and biology of mucopolysaccharides. Am. J. Med., 47: 664-672.

Nelson, M. M., C. W. Asling and H. M. Evans 1952 Production of multiple congenital abnormalities in young by maternal pteroylglutamic acid deficiency during pregnancy. $J$. Nutr., 48: 61-79.

Nelson, M. M., H. V. Wright, C. W. Asling and H. M. Evans 1955 Multiple congenital abnormalities resulting from transitory deficiency of pteroylglutamic acid during gestation in the rat. J. Nutr., 56: 349-369.

Patten, B. M., T. C. Kramer and A. Barry 1948 Valvular action in the embryonic heart by localized apposition of endocardial masses. Anat. Rec., 102: 299-311.

Runner, M. N. 1965 General mechanisms of teratogenesis. In: Teratology: Principles and Techniques. J. G. Wilson and J. Warkany, eds. Univ. of Chicago Press, Chicago, pp. 95-103.

Turbow, M. M., and J. G. Chamberlain 1968 Direct effects of 6-aminonicotinamide on the developing rat embryo in vitro and in vivo. Teratology, 1: 103-108.

Walker, B. E. 1961 The association of mucopolysaccharides with morphogenesis of the palate and other structures in mouse embryos. J. Embryol. Exp. Morph., 9: 22-31.

Wilson, J. G. 1960 Experimental production of congenital cardiac defects. In: Congenital Heart Disease. American Association for the Advancement of Science, Washington, D. C., pp. 65-82. 